\title{
Infectious diseases of economic importance: Molecular biological characteristics of foot-and-mouth disease viruses collected in Tanzania from 1967 to 2009
}

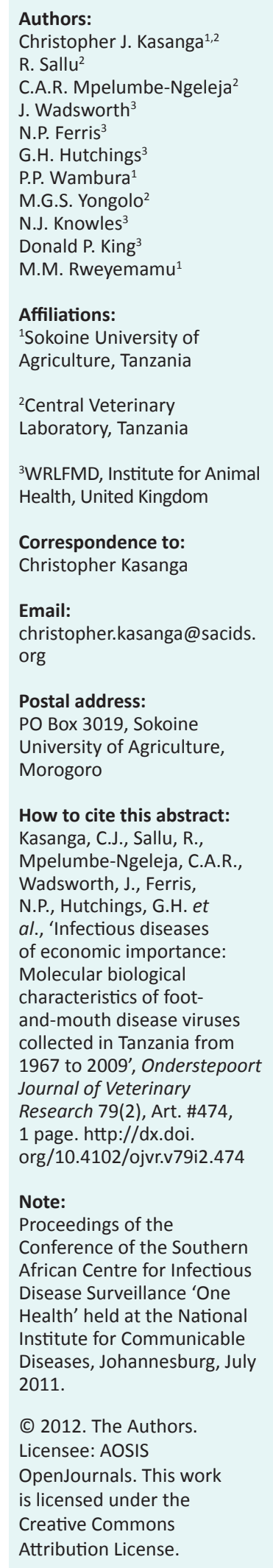

Foot-and-mouth disease (FMD) is endemic in Tanzania. Since the first reports in 1954, FMD has caused significant economic losses in the country due to mortality and morbidity of livestock and costs associated with controlling the disease. The aim of this study was to review the serotype and genetic relationships of the FMD virus (FMDV) recovered from outbreaks in Tanzania, and compare them with viruses detected from elsewhere in the sub-Saharan region. At the World Reference Laboratory for foot-and-mouth disease (WRLFMD), a total of 106 FMD viruses have been isolated from samples collected between 1967 and 2009 from northern, southern, eastern and central parts of Tanzania. The presence of FMDV was determined by laboratory methods such as VI, CF, antigen ELISA and RT-PCR. Phylogenies of VP1 sequences were determined by the Neighbour-joining method. Foot-and-mouth disease virus SAT1 was the most frequent serotype $(46.2 \% ; n=49)$ isolated in Tanzania followed by $\mathrm{O}(26.4 \% ; n=27)$, A $(14.1 \% ; n=15)$ and SAT $2(11.3 \% ; n=13)$. Genotyping showed that type $O$ viruses fell into either the EAST AFRICA 1 (EA-1) or EA-2 topotypes, type A's into the AFRICA topotype (genotype I), type SAT 1's into topotype I and type SAT 2's into topotype IV. This study reveals that serotypes A, O, SAT1 and SAT2 cause FMD outbreaks in Tanzania. Recent samples from outbreaks in 2008, 2009 and 2010 have been typed as serotypes A, O, SAT1 and SAT2. Phylogenetic analysis of FMDV isolates from Tanzania showed that they are genetically related to lineages and topotypes from West and East Africa. In Tanzania, lack of comprehensive animal movement records and inconsistent vaccination programs make it difficult to determine the exact source of FMD outbreaks or to trace the transmission of the disease over time. Therefore, further collection and analysis of samples from domestic and wild animals, together with improved local epidemiological investigation of FMD outbreaks is required to elucidate the complex epidemiology of FMD in the sub-Saharan region. 